\section{Die HPV-Impfung für Jungen}

Humane Papillomaviren erzeugen die
häufigsten übertragenen sexuellen Infek-
tionen in Deutschland und weltweit.
Neben Gebärmutterhalskrebs können sie
weitere Tumoren des Anogenitale und
des Oropharynx hervorrufen und sind
Auslöser für Condylome. Die derzeitige
von der Ständigen Impfkommission (STI-
KO) empfohlene Praxis, lediglich Mäd-
chen zwischen 9 und 14 Jahren zu impfen
(Stand: 8/2015), ist nach Ansicht der
DGU und des BDU nicht mehr zeitgemäß.

Sie berücksichtige auch nicht die vielfachen Lebenswelten sexuell aktiver Menschen in unserem Land. „Der oft angemerkte Herdenschutz durch die Impfung der Mädchen greift ja bereits dann nicht, wenn wir es mit jungen Männern zu tun haben, die Sex mit Männern haben (MSM)“, so DGU-Präsident Prof. Kurt Mil- ler. Im Sinne eines umfassenden Schutzes unabhängig von der sexuellen Orientierung empfehlen DGU und BDU nun gemeinsam „Impft auch die Jungen!“.

HP-Viren sind für praktisch alle Gebärmutterhalskrebserkrankungen verantwortlich, damit auch für über 100000 vermeidbare Konisationen und rund 3000 weibliche Todesfälle pro Jahr in Deutschland. „Ein Argument für die alleinige Impfung der Mädchen war bisher immer, auf diese Weise die HPV-Last bei sexuell aktiven jungen Frauen so stark abzusenken, dass sich die jungen Männer als Sexualpartner der geimpften Frauen ebenfalls nicht mehr anstecken können. Dieser Herdenschutz funktioniert allerdings nur, wenn über die HPV-Impfung mehr als $85 \%$ der jungen Mädchen erfasst würden“, so Miller weiter. „Da der Penis der HauptTransmitter für HPV darstellt, ist der Verzicht auf die Jungenimpfung fahrlässig.“

In der Realität liegen jedoch die Impfquoten der Mädchen für die letzten Jahre mit weniger als $40 \%$ deutlich unterhalb der erforderlichen Menge. Diejenigen, die indes einen der derzeit verfügbaren multivalenten Impfstoffe erhalten hatten, zeigen deutlich weniger Condylomata und Krebsvorstufen, wie aus deutschen, dänischen und australischen Studien hervorgeht. Ganz ähnliche Daten finden sich für geimpfte Jungen aus den USA und Australien als Vorreiter der HPV-Impfung für diese Zielgruppe.

Die HPV-Impfung ist sicher und schützt effektiv vor der Infektion mit einigen der Hochrisiko-HP-Viren. Die höchste Wirksamkeit zeigt die Impfung vor dem ersten Geschlechtsverkehr, jedoch steht inzwischen fest, dass auch bei sexuell erfahrenen Erwachsenen die Folgeerkrankungen nach erfolgter Infektion wie auch die Rezidive bei bereits manifesten und behandelten Zervixkarzinomen signifikant reduziert werden. „Die international vorliegenden wissenschaftlichen Daten stützen unsere gemeinsame Empfehlung für eine HPV-Impfung bei Jungen“, sagt Prof. Oliver Hakenberg, Generalsekretär der DGU. BDU-Präsident Dr. Axel Schroeder ergänzt: „Mit der Jungensprechstunde beim Urologen verfügen wir inzwischen zudem über eine geeignete Versorgungsstruktur, um möglichst viele Jungen zu erreichen, sie über die HPV-Impfung aufzuklären und letztlich auch zu impfen.“

Nach einer Pressemitteilung (DGU/BDU) 